\title{
Conditions affecting the pericardium
}

Melanie Humphreys, MA, BSc (Hons), ENB 124, RGN, ONC, Senior Lecturer, Post-registration Studies, School of Health, University of Wolverhampton, UK

e-mail: M.Humphreys@wvl.ac.uk

Key words: acute pericarditis cardiac tamponade clinical features ECG changes pericardial effusion

\section{INTRODUCTION}

Conditions of the pericardium can be associated with electrocardiographic (ECG) abnormalities. Whilst the ECG per se, is neither a sensitive or specific tool for diagnosing such conditions, an awareness of the ECG abnormalities associated with the conditions of pericarditis, pericardial effusion and cardiac tamponade may offer supportive evidence in conjunction with the patient's clinical assessment towards accurate diagnosis. This article will review both the clinical picture and the common ECG findings of these three conditions that affect the pericardium.

\section{BACKGROUND}

The pericardium is a double-walled, fibroserous, inelastic sac that encircles the heart. The outer fibrous layer of the pericardium is attached to the great vessels, the sternum, and diaphragm. This fibrous layer of the pericardium is very resistant to distension and helps to prevent dilation of the heart. The inner serous layer consists of a visceral and parietal portion. The visceral portion, also known as the epicardium, covers the entire heart and great vessels and folds over to form the parietal pericardium. The parietal portion also lines the fibrous pericardium (Gavaghan, 1998).

The pericardium appears to serve three functions:

1. It fixes the heart within the mediastinum and limits its motion

2. It prevents extreme dilatation of the heart during sudden rises of intracardiac volume (e.g. acute mitral regurgitation),

3. It may function as a barrier to limit the spread of infection from the adjacent lungs.

However, people with complete absence of the pericardium (either congenital, or surgically removed) generally do fine without it, casting doubt on its actual physiologic importance (Lilly, 1993).

The pericardial cavity is a potential space between the parietal and visceral pericardium. It normally contains $30 \mathrm{ml}$ to $50 \mathrm{ml}$ of serous fluid that acts as a lubricant to decrease friction as the heart contracts and relaxes. The pericardial cavity can hold up to one litre of fluid in some chronic diseases without compromising the heart (Porth, 1994). If the build up of fluid occurs gradually, the pericardium can stretch without affecting the heart. If the fluid accumulates rapidly, however, even small amounts (i.e. $50 \mathrm{ml}$ to $100 \mathrm{ml}$ ) may profoundly increase this pressure and result in lifethreatening illness. With conditions affecting the pericardium, it is vital to consider the overall clinical picture in conjunction with the patient's electrocardiograph (ECG), in order that an accurate diagnosis can be made.

\section{ACUTE PERICARDITIS}

The most common affliction of the pericardium is acute pericarditis, which refers to inflammation of its layers. Many disease states can produce this syndrome; however, there are two forms of pericarditis associated with acute myocardial infarction (MI). The early form occurs within the first few days of $\mathrm{MI}$, affecting $10-15 \%$ of patients (Connaughton, 2001; Dracup, 1995). It likely results from epicardial inflammation (visceral layer) extending from the injured myocardium, and is therefore most common in patients with transmural infarctions (Tofler et al., 1989).

The second form is Dressler's syndrome, which develops 2 weeks to several months following an acute MI. The cause is unknown, but it is thought to be of autoimmune origin, possibly directed against antigens released from necrotic myocardial cells (Lilly, 1993). It can be associated with more generalised pericarditis and a loud pericardial friction rub together with fever and elevated erythrocyte sedimentation rate (Kossowsky et al., 1981).

The acute inflammatory process of pericarditis is characterised by three stages: (1) local vasodilation with transudation of protein-poor, cell-free fluid into the pericardial space, (2) increase of vascular permeability, with leak of protein into the pericardial space, and (3) leukocyte exudation, initially by neutrophils, followed later by mononuclear cells. The leukocytes are of critical importance because they can help to contain or eliminate the offending infectious or autoimmune agent. However, metabolic products released by these cells may prolong inflammation, cause pain and local cellular damage, and mediate somatic symptoms such as fever. Therefore, the immune response to pericardial injury may significantly contribute to tissue damage and symptoms (Lilly, 1993; Tortora and Anagnostakos, 2000).

It is important to distinguish pericarditic pain from recurrent myocardial ischaemia (Cheitlin, 1997). The history will usually 
be helpful. Pericarditis is unlikely in the first 24 hours post MI (Connaughton, 2001). Further indicators of pericarditis include a positional or inspiratory component to the pain or radiation to the left shoulder or scapula. An audible rub is diagnostic, especially if the patient is lying flat (see below). A transient pericardial rub may be detected in $10-15 \%$ of patients with acute MI. Whilst pericarditis can occur in anterior or inferior infarction, a pericardial friction rub usually accompanies pericarditis only in anterior MI (Thompson, 1997). Some claim that the prognosis following acute $\mathrm{MI}$ is not affected by the presence of pericarditis (Cheitlin, 1997). However, others have argued that patients with pericarditis have a worse outcome, but this is probably due to the associated extensive infarction rather than the pericarditis per se (Thompson, 1997). Thus, while pericarditis itself is usually benign and selflimiting it may be a marker of a relatively large infarction. It has been asserted that patients with pericarditis have lower ejection fractions and a higher subsequent incidence of heart failure (Tofler et al., 1989; Wall, 1990). However, in the main, the pericarditis usually disappears spontaneously within a few days and seldom causes serious problems (Dracup, 1995).

\section{Clinical features}

1. Chest pain - severe, often localised to the retrosternal area and left precordium; may radiate to the back and ridge of the left trapezius muscle, typically pleuritic and positional (aggravated by inspiration and coughing: sitting and leaning forward often lessen the discomfort).

2. Friction rub - produced by the movement of the inflamed pericardial layers against one and another. Auscultation of the rub is best heard using the diaphragm of the stethoscope with the patient leaning forward, while exhaling. Characteristically, the pericardial rub is evanescent, so that it may come and go from one examination to the next (Spodick, 1989).

\section{ECG identification}

The ECG is abnormal in $90 \%$ of patients with acute pericarditis, and helps to distinguish it from other forms of cardiac disease such as acute MI (Dracup, 1995). The most important ECG pattern, which reflects inflammation of the adjacent myocardium, consists of diffuse ST-segment elevation, in almost all of the ECG leads, but it is especially seen in I, II, aVL, aVF \& V3-V6. In contrast ST segment depression always occurs in aVR and usually in V1 (Spodick, 1989). The elevation is typically presented as J point elevation (or notching) with concave upward ST segment elevation (often described as scooping) (Connaughton, 2001). The amount of elevation is variable and can be quite high, up to 4 to $5 \mathrm{~mm}$. There tends to be diffuse elevation because of the irritation to the whole of the pericardium, which causes a net positivity of the epicardium (reflected electrocardiographically as ST elevation) (Wagner, 1994). The T waves usually remain upright in those leads exhibiting ST segment elevation (Braunwald, 1992). These abnormalities are in contrast to the ECG of acute $\mathrm{MI}$, in which the ST segments are elevated only in the leads overlying the region of the infarction (Lilly, 1993; Connaughton, 2001).

PR segment depression is often evident, reflecting abnormal atrial repolarisation related to the inflammatory process (Woods, et al., 1995; Garcia \& Holtz, 2001). Late presentations of pericar-

\section{Box 1}

Undertake a systematic analysis of the following 12-lead ECG

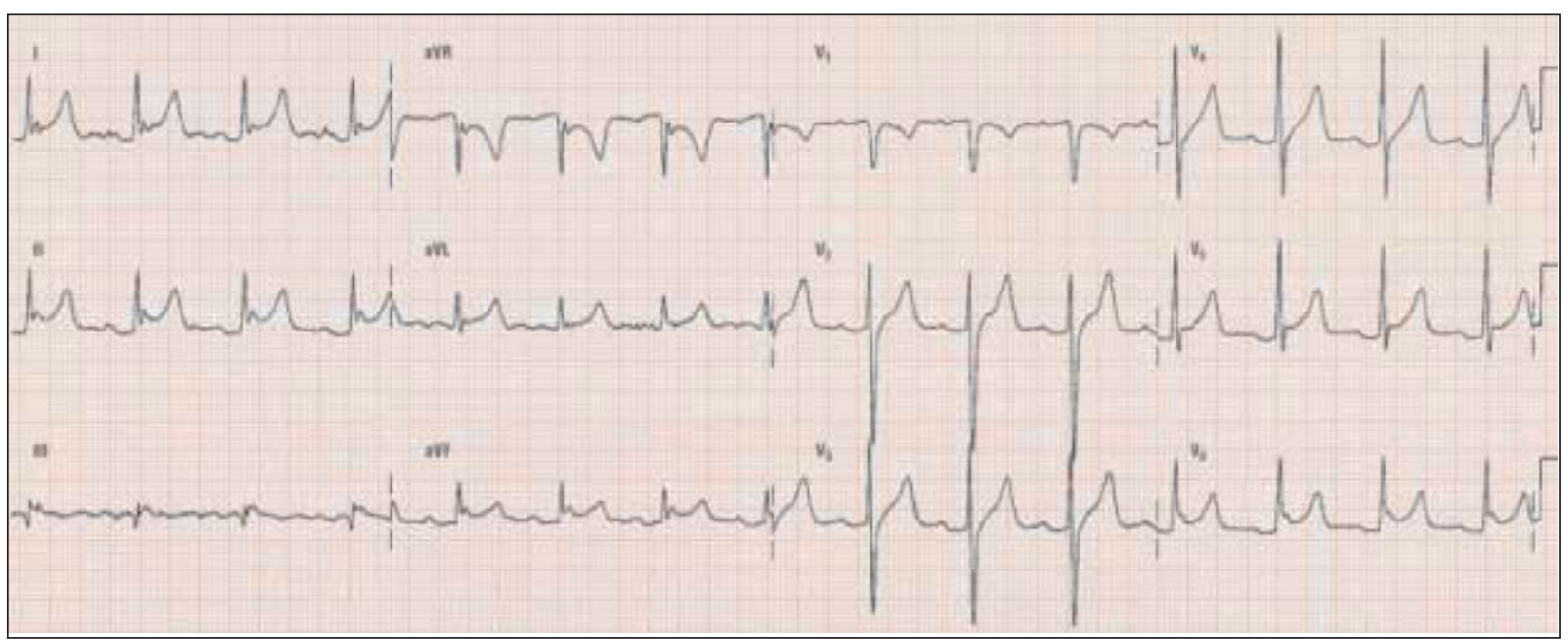

\section{COMMENTARY}

The 12-lead ECG shows the following features:

* Sinus rhythm, rate 85 bpm

* Rhythm - regular

* P waves - normal, PR interval - 0.18 sec., PR segment - depressed $1.0 \mathrm{~mm}$ leads I, II, aVF, \& V5-V6

* Normal QRS axis

* QRS notching at the J point in inferior leads, and V5-V6

* ST segments - elevated in leads II, III, aVF, V3-V6, with concave upwards appearance (scooping), aVR shows ST depression.
This ECG shows all the criteria for acute pericarditis:

1. Diffuse ST segment elevations, which are scooped upwardly concave

2. ST depression in aVR

3. PR depression

4. Notching at the end of the QRS complexes.

When applying this to the patient's clinical history and presentation a diagnosis of Dressler's syndrome was made, this resolved over a few days. 
ditis could include generalised $\mathrm{T}$ wave inversion in most leads; this may persist indefinitely, and presents a picture difficult to distinguish from diffuse myocardial ischaemia (Braunwald, 1992; Woods, 1995).

The critical aspect of correctly diagnosing pericarditis however, remains with the history and physical examination. These should be consistent with pericarditis; if they match the ECG findings, then the diagnosis is clear.

\author{
Other common causes of pericarditis \\ * Viral \\ * Malignancy \\ * Uraemia \\ * Tuberculosis (TB) \\ * Connective tissue disease \\ Hypothyroidism (Jenkins \& Gerred, 1999).
}

Treatment usually focuses on rest and pain relief through the administration of non-steroidal anti-inflammatory therapy (Woods et al., 1995).

\section{PERICARDIAL EFFUSION}

Pericardial effusion is the abnormal accumulation of more than $50 \mathrm{ml}$ of fluid in the pericardial sac (Spodick, 1997). An effusion may be non-compressive or compressive. A non-compressive pericardial effusion does not interfere with normal cardiac function and may be clinically silent. A compressive pericardial effusion, however, precipitates a sudden increase in intrapericardial pressure with subsequent development of cardiac tamponade (Woods et al., 1995).

Three factors determine whether a pericardial effusion remains clinically silent, or whether symptoms of cardiac compression ensue: (1) the volume of fluid, (2) the rate at which the fluid accumulates, and (3) the compliance characteristics of the peri- cardium.

The intrapericardial pressure determines the haemodynamic consequences of pericardial effusion. Because the pericardium is a stiff structure, the relationship between its internal volume and pressure is not linear. At the low volumes normally present within the pericardium, a small increase in volume leads to only a small rise in pressure. However, when the intrapericardial volume expands beyond a critical level a dramatic increase in pressure is incited by the non-distensible sac. At this point, a meagre increase in volume translates into an enormous compressive force on the heart (Braunwald, 1992; Lilly, 1993). Indeed, the sudden increase of pericardial volume by as little as 100 to $250 \mathrm{ml}$, as may occur with intrapericardial haemorrhage, results in marked elevation of pericardial pressure, and severe cardiac chamber compression. Even smaller amounts of fluid may cause marked elevation of pressure if the pericardium is pathologically non-compliant and stiff, as may occur in the presence of fibrosis of the sac. In contrast, if the pericardial effusion accumulates slowly, over weeks to months, the pericardium does gradually stretch, and much larger volumes are accommodated at lower pressures (Battle \& LeWinter, 1990; Thompson, 1997). Indeed, such a pericardium can accommodate 1 to 2 I without significant elevation of intrapericardial pressure.

\section{Clinical features}

The patient with a large pericardial effusion may be asymptomatic, may complain of a dull constant ache in the left side of the chest, or present with symptoms of cardiac compression (see cardiac tamponade below). The presence of pericardial fluid insulates the heart from the chest wall, and the heart sounds will be muffled. A friction rub that had been present during the acute phase of pericarditis may disappear if an effusion widely separates the inflamed layers. Dullness to percussion of the left lung over the angle of the scapula may occur, known as 'Ewart's sign', and is due to compressive atelectasis by the large pericardial sac (Lilly, 1993).

\section{Box 2}

Consider the following two rhythm strips

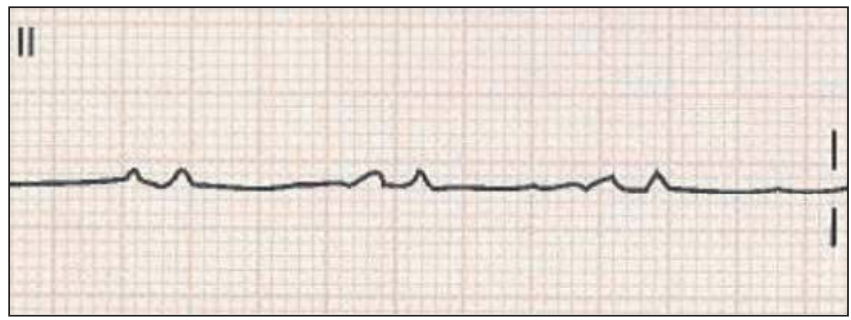

\section{COMMENTARY}

These two leads were taken from a 12-lead ECG, where all complexes were similarly small. The rhythm strips show the following features:

$$
\begin{aligned}
& \text { The rhythm is sinus rhythm, rate } 90 \mathrm{bpm} \\
& \text { P waves are evident and almost equal to QRS amplitude } \\
& \text { The amplitude of the QRS in Lead II is } 0.15 \mathrm{mV} \text {, and } \\
& 0.1 \mathrm{mV} \text { in V4 } \\
& \text { T waves are flattened. }
\end{aligned}
$$

With a large pericardial effusion, this dampening effect may be seen. It is important to realise that the ECG is only accurate if the electrocardiogram is performed in a standardised fashion; this would include both paper speed (vertical lines) and voltage. It is imperative that these are assessed prior to analysis. It is the voltage (horizontal lines) in particular that enables one to make judgements regarding the amplitude

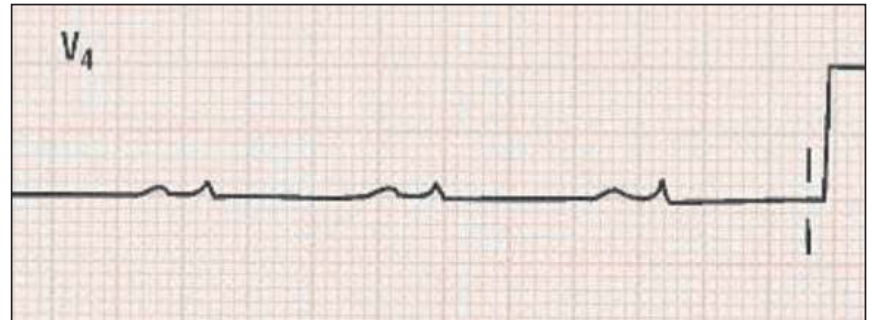

of the QRS. The standard signal is a $1 \mathrm{mV}$ deflection $10 \mathrm{~mm}$ high. With this standard, $1 \mathrm{~mm}$ in height equals $0.1 \mathrm{mV}$. This is known as Std 1 (this is evident in the terminal portion of the rhythm strip recorded in $\mathrm{V}_{4}$ ). Therefore, calculations regarding amplitude can be made and significance applied. A voltage in all of the limb leads of less than $5 \mathrm{~mm}$ is abnormal waves less than $10 \mathrm{~mm}$ high in all of the precordials are also abnormal (Garcia and Holtz, 2001). The amplitude of this ECG is extremely small and was caused because of a large pericarditic effusion in which 11 of strawcoloured fluid was aspirated. 


\section{ECG identification}

As the pericardial fluid accumulates, the ECG may exhibit reduced voltage of the complexes because of the insulation effect of the surrounding fluid. In the presence of extremely large effusions, the height of the QRS complex may vary from beat to beat (electrical alternans); this results from a constantly changing electrical axis, as the heart swings within the large pericardial volume (Lilly, 1993; Wagner, 1994). There may be flattening of the T waves, while normal $P$ wave voltage remains (Woods et al., 1995).

As with pericarditis, the critical aspect of correctly diagnosing pericardial effusion remains with a consistent history and physical examination, in conjunction with key ECG findings.

It is important to remember that an ECG can meet limb lead voltage without meeting precordial criteria. This occurs because the precordial leads are directly overlying the heart on the chest wall. A thin person with an effusion will have larger precordial leads than a heavier person with an effusion.

Other causes of small QRS complexes (dampeners):

Obesity - excess adipose tissue

Hypothyroidism - amyloid deposits

Localised left pleural effusion - voltage in certain

leads (typically V5 and V6)

- Women have smaller amplitude than men do

- Large breasts, and/or silicone breast implant

- Elderly have smaller amplitude than young people do

[ L Limb leads are smaller than precordials because they are further away from the heart.

(] Chronic obstructive airways disease

[ Acute pulmonary embolus

(Jenkins and Gerred, 1999; Garcia and Holtz, 2001).

The prognosis and treatment of pericardial effusion depend on its haemodynamic significance and the presence and nature of any underlying systemic illness. Pericardial drainage is indicated for signs of cardiac compression and may be accomplished by pericardiocentesis (Kirkland and Taylor, 1992).

\section{CARDIAC TAMPONADE}

At the opposite end of the spectrum from the asymptomatic pericardial effusion is cardiac tamponade, the condition in which pericardial fluid accumulates under high pressure. Because of the tense pericardial fluid, the heart is compressed, and the diastolic pressure within each chamber becomes elevated and equal to the compressive pericardial pressure (Lilly, 1993; Thompson, 1997). Because filling of the heart chambers is impaired, the systemic and pulmonary venous pressures become elevated, as normal return of blood to the heart cannot be accommodated. The increase of systemic venous pressure results in signs of right-sided heart failure are distension of the jugular veins, oedema, and hepatomegaly. Decreased atrial filling leads to inadequate ventricular filling, reduced cardiac output, and potential circulatory collapse (Tortora and Anagnostakos, 2000). Pulsus paradoxus, in which arterial blood pressure during expiration exceeds arterial pressure during inspiration by more than $10 \mathrm{mmHg}$, is a key indicator of cardiac tamponade. Normally, inspiration has little effect on cardiac flow or volume. A 'water bottle' cardiac silhouette on a chest x-ray, dyspnoea on exertion, dull chest pain, and muffled heart sounds are other signs of cardiac tamponade (McCance, 1994). In addition, reduced filling of the ventricles during diastole decreases blood available for systolic stroke volume, and the cardiac output declines. Failure to decompress the heart leads to inadequate perfusion of vital organs, shock and ultimately death (Resuscitation Council, UK, 2001). Any episode of acute pericarditis can progress to cardiac tamponade. Acute haemorrhage into the pericardium is an important result of left ventricular free wall rupture following a MI. This occurs in $1-4 \%$ of post-MI patients and is almost always rapidly fatal, frequently presenting as chest pain, haemodynamic collapse and pulseless electrical activity (PEA) (Connaughton, 2001). Rupture of the ventricular wall may occur at any time during hospitalisation for $\mathrm{MI}$, but the highest incidence is within the first 5 to 7 days. It is more likely to occur in women, in patients over 50 years of age, and in patients who have been hypertensive prior to the onset of their MI (Dracup, 1995).

\section{Clinical Features}

Cardiac tamponade should be suspected in any patient with known pericarditis, pericardial effusion, or chest trauma who develops signs and symptoms of systemic vascular congestion and decreased cardiac output. The key physical findings include (1) jugular venous distension, (2) systemic hypotension, and (3) a small quiet heart on physical examination, due to the insulating effects of the effusion. Other signs include sinus tachycardia and pulsus paradoxus. Dyspnoea and tachypnoea reflect pulmonary congestion, as well as decreased oxygen delivery to peripheral tissues.

\section{ECG identification}

If the tamponade has developed slowly, over weeks or months, then the ECG will share the characteristics of pericardial effusion (Wagner, 1994). In the acute emergency situation, it would be inappropriate to record a 12-Lead ECG, as resuscitation including removal of the high-pressure pericardial fluid (pericardiocentesis), is the only measure that has the potential to reverse this life-threatening condition (Resuscitation Council, UK, 2001).

\section{CONCLUSION}

Knowledge of conditions that affect the pericardium facilitates the critical care nurses understanding of those patients at risk. Acute pericarditis can mimic angina pectoris, especially when secondary to an acute MI. Therefore, it is very important for the critical care nurse to evaluate the pain descriptors and associated symptoms carefully. Astute observations for signs and symptoms of cardiac tamponade are critical to expedite treatment for this potentially life-threatening complication (Woods et al., 1995; Resuscitation Council, UK, 2001). Consequently, through assessment of presenting signs and symptoms and patient history, in conjunction with 12-lead ECG evaluation, early detection and prompt treatment will be made. A greater understanding of the pathophysiological processes involved can only serve to enhance individualised care delivery within the acute care setting.

\section{REFERENCES}

Battle RW, Lewinter MM. (1990). The evaluation and management of pericardial disease. Current Opinion in Cardiology; 5: 331-9.

Braunwald E. (1992). A Textbook Of Cardiovascular Medicine. (4th edn.). Philadelphia:WB Saunders.

Cheitlin MD. (1997). ACC/AHA Guidelines For The Clinical Application Of Echocardiography. A Report Of The American College Of

CardiologyAmerican Heart Association Task Force On Practice

Guidelines (Committee On Clinical Application Of Echocardiography). Developed in collaboration with the American

Society of Echocardiography. Circulation; 95: 1686-1744.

Connaughton M. (2001). Evidence-Based Coronary Care. London: Churchill Livingstone. 
Garcia TB, Holtz NE. (2001). 12-lead ECG: the art of interpretation. London: Jones and Bartlett Publishers.

Gavaghan M. (1998). Cardiac anatomy and physiology: a review. Aron Journal; 67: 802-822.

Jenkins RD, Gerred SJ. (1999). ECGs by example. London: Churchill Livingstone. Kirkland LL, Taylor RW. (1992). Pericardiocentesis. Critical Care Clinics; 8: 699712 .

Kossowsky W, Lyon AF, Spain DM. (1981). Reappraisal of the post myocardial infarction dressler's syndrome. American Heart Journal; 102: 954-6.

Lilly LS. (1993). Pathophysiology of Heart Disease: A collaborative Project of

Medical Students and Faculty. London: Lea \& Febiger.

Mccance KL, Heuther SE. (1994). Pathophysiology: the biologic basis for disease in adults and children. (2nd edn.). St Louis: Mosby Year Book Inc.

Porth CM. (1994). Pathophysiology: concepts of altered health states. (4th edn.). Philadelphia: Lippincott.

Resusciation Council (UK). (2000). Advanced life support course (4th edn.). London: Resusciation Council (UK) and European Council.
Smith It, Kampine JP. (1990). Circulatory Physiology - the London: Williams \& Wilkins.

Spodick DH. (1989). Pericarditis, pericardial effusion, cardiac constriction. Critical Care Clinics; 5: 455-76.

Spodick DH. (1997). Pericardial Disease. JAMA; 278: 704

Tofler GH, Muller JE, Stone PH, Willich SN, Davis VG, Poole WK, Braunwald E. (1989). Pericarditis in acute myocardial infarction: clinical significance. American Heart Journal; 117: 86-

Tortora GJ, Anagnostakos NP. (2000). Principles of Anatomy edn.). New York: Harper Collins.

Wagner GS. (1994). Marriott's Practical Electrocardiography. Williams \& Wilkins.

Wall TC. (1990). Usefulness of a pericardial friction rub after during acute myocardial infarction in predicting amount The TAMI Study Group. American Journal of Cardiology;

Woods SL, Froelicher ESS, Halpenny CJ, Underhill-Motzer S. Nursing. (3rd edn.). Pennsylvania: Lippincott Company. 